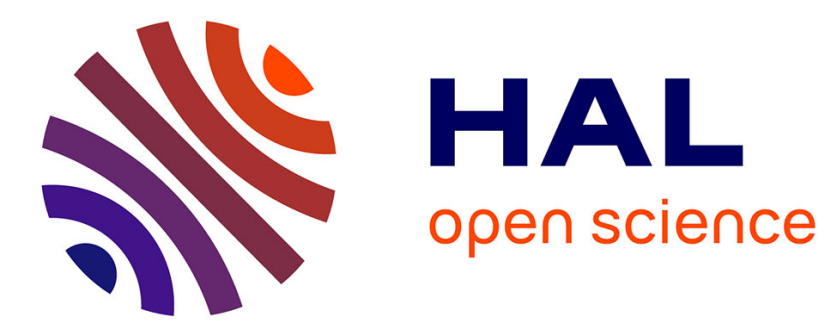

\title{
Micromechanics of Surface-Grafted Hyaluronic Acid Gels
}

Guy Ladam, Laurent Vonna, Erich Sackmann

\section{To cite this version:}

Guy Ladam, Laurent Vonna, Erich Sackmann. Micromechanics of Surface-Grafted Hyaluronic Acid Gels. Journal of Physical Chemistry B, 2003, 107 (34), pp.8965-8971. 10.1021/jp0272872 . hal02337111

\section{HAL Id: hal-02337111 \\ https://hal.science/hal-02337111}

Submitted on 29 Oct 2019

HAL is a multi-disciplinary open access archive for the deposit and dissemination of scientific research documents, whether they are published or not. The documents may come from teaching and research institutions in France or abroad, or from public or private research centers.
L'archive ouverte pluridisciplinaire HAL, est destinée au dépôt et à la diffusion de documents scientifiques de niveau recherche, publiés ou non, émanant des établissements d'enseignement et de recherche français ou étrangers, des laboratoires publics ou privés. 


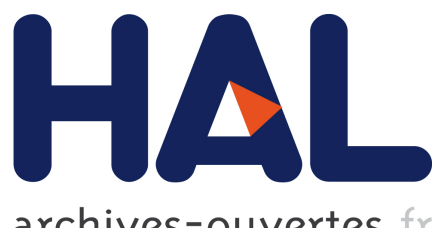

archives-ouvertes

\title{
Micromechanics of Surface-Grafted Hyaluronic Acid Gels
}

\author{
Guy Ladam, Laurent Vonna, Erich Sackmann
}

\section{To cite this version:}

Guy Ladam, Laurent Vonna, Erich Sackmann. Micromechanics of Surface-Grafted Hyaluronic Acid Gels. Journal of Physical Chemistry B, American Chemical Society, 2003, 107 (34), pp.8965-8971. 10.1021/jp0272872 . hal-02337111

\section{HAL Id: hal-02337111 \\ https://hal.archives-ouvertes.fr/hal-02337111}

Submitted on 29 Oct 2019

HAL is a multi-disciplinary open access archive for the deposit and dissemination of scientific research documents, whether they are published or not. The documents may come from teaching and research institutions in France or abroad, or from public or private research centers.
L'archive ouverte pluridisciplinaire HAL, est destinée au dépôt et à la diffusion de documents scientifiques de niveau recherche, publiés ou non, émanant des établissements d'enseignement et de recherche français ou étrangers, des laboratoires publics ou privés. 


\title{
Micromechanics of Surface-Grafted Hyaluronic Acid Gels
}

\author{
Guy Ladam,* Laurent Vonna, and Erich Sackmann \\ Lehrstuhl für Biophysik, E22, Technische Universität München, James-Franck-Strasse, \\ D-85748 Garching, Germany
}

Received: October 23, 2002; In Final Form: April 15, 2003

\begin{abstract}
To design models of tissue surfaces, films of soft gels of hyaluronic acid (HA) (a ubiquitous constituent of the extracellular matrix) are covalently grafted to glass substrates functionalized with aminosilane monolayers. Gelation is achieved by coupling of carboxyl groups of HA through carbodiimide. The elasticity of the gel is controlled through the HA concentration. The magnetic bead microrheometry and colloidal bead deformation field mapping techniques are applied to measure the surface viscoelastic moduli and the effective Young moduli of the HA gel as a function of the gel density. For this purpose, magnetic beads and nonmagnetic beads are coupled to the surface of the HA films. Soft homogeneous films exhibiting Young elastic moduli between 3 and $250 \mathrm{~Pa}$ were generated. The shear deformation field induced by tangential force pulses applied to the magnetic beads is measured and analyzed in terms of the theory of elastic deformation of half-spaces by local forces. An unconventional vortex-deformation field is observed for gel film thicknesses of several hundred micrometers, which is attributed to the nonlinear elasticity of the gels. We finally show that amoebalike cells of the slime mold Dictyostelium discoideum spontaneously adhere to the HA film, while fibroblast adhesion can be mediated through coupling fibronectin to the surface.
\end{abstract}

\section{Introduction}

The present work deals with the design of soft films of hyaluronic acid of variable viscoelastic impedance on solids, which may serve as models of the extracellular matrix for studies of cell functions (such as locomotion and adhesion) on biofunctional surfaces. Hyaluronic acid (also abbreviated as HA in the following) is a highly negatively charged polysaccharide composed of disaccharide repeat units (consisting of glucuronic acid and $N$-acetylglucosamine). It is a major constituent of many types of tissue. Examples are skin (where it serves as matrix for the cells such as keratinocytes) and the vitreous body of the eyeball. Owing to the unique viscoelastic properties of swollen HA, it also plays a key role as constituent of the lubricating synovial fluid of joints. Last but not least, HA is a regulator of cell adhesion ${ }^{1,2}$ and as such plays an important role during embryonic development, ${ }^{3}$ for instance, as an entropic springlike buffer between mesenchyme cells. For this purpose, many cells exhibit receptors with lectin-like headgroups (such as CD44), and HA thus mediates cell-cell attraction over long distances but prevents strong adhesion by the other cell receptors. Besides its biological importance, HA is also of interest for studies of fundamental physicochemical properties of solutions and networks of polyelectrolytes. HA is a giant macromolecule exhibiting molecular weights of up to $4 \times 10^{6}$ Da corresponding to $10^{4}$ repeat units and contour lengths on the order of $10 \mu \mathrm{m}$. At neutral $\mathrm{pH}$, the glucuronic acid is dissociated and the linear charge density is $\rho \approx 1 \mathrm{e} \mathrm{nm}-1$.

In the present work, we were mainly concerned with the preparation of 200-2000 $\mu \mathrm{m}$ thick solid-supported and homogeneous HA gels, which are covalently grafted to the substrate while the viscoelastic impedance is adjusted through the HA concentration. In an accompanying study, we developed a

* To whom correspondence should be addressed. E-mail address: Guy.Ladam@univ-rouen.fr. technique to deposit ultrathin HA films ( $200 \mathrm{~nm}$ thick) on glass substrates, which is based on the anchorage of the polymer to fluid-supported membranes through hyaluronic acid binding proteins coupled to lipid molecules. ${ }^{4}$

Moreover, we show how the viscoelastic modulus and the Poisson ratio of polymer films can be measured by magnetic tweezer microrheometry in combination with the deformation field mapping technique. For this purpose, the surface of the HA layers is decorated with magnetic beads used as force transducers and nonmagnetic colloidal beads acting as indicators of the deformation field induced by the tangential deflection of a force transducer. The beads are covalently coupled to the polymer surface. The Poisson ratio and the Young modulus are obtained by analyzing the deformation field in terms of the theory of deformation of a half-space by a tangential point force acting on its surface. ${ }^{5}$ In submillimeter thick films local tangential forces induce vortex-like deformation fields, which are attributed to nonlinear viscoelastic effects.

In preliminary experiments, we show that mammalian cells (e.g., fibroblasts) can be immobilized on the HA surface by covering the surface with fibronectin and that cells of the slime mold Dictyostelium discoideum adhere directly on the HA gel surface.

\section{Materials and Methods}

Chemicals. Bacterial hyaluronic acid sodium salt from Streptococcus zooepidemicus (HA, 1.24 MDa, ref H-9390) was a product from Sigma (Germany). $N$-[3-(Dimethylamino)propyl]-3-ethylcarbodiimide hydrochloride (EDC, ref 22981) and $N$-hydroxysuccinimide (NHS, ref 24500) were purchased from Pierce (Germany). 4-(2-Hydroxyethyl)piperazine-1-ethanesulfonic acid buffer (HEPES, ref 54457) and absolute ethanol (ref 32205) were purchased from Fluka (Germany). N-[6(Aminohexyl)aminopropyl]-trimethoxysilane (AHAPTMS, 95\%, ref 1E-8708) was a product from Gelest (Tullytown, PA). All 


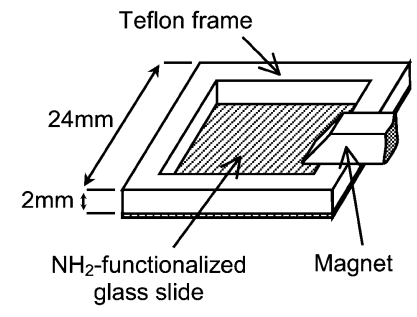

Figure 1. Measuring cell used to prepare "bulk" HA gels, indicating the position of the sharp edge of the soft iron core of the magnet.

of the chemicals of commercial origin were used without further purification. All solutions were prepared and all cleaning steps were performed with ultrapure water (Milli-Q system, Millipore, Bedford, MA).

Probes and Markers. Tosyl-activated superparamagnetic Dynabeads M450 (Ø $4.5 \mu \mathrm{m}$, density approximately $1.5 \mathrm{~g} \mathrm{~cm}^{-3}$, ref 140.03) were purchased from Dynal (Germany). Polybead polystyrene amino-functionalized microspheres $(\varnothing 0.5 \mu \mathrm{m}$, ref 07763) were purchased from Polysciences (Warrington, PA). Both types of beads were washed and diluted prior to use.

Preparation of Hyaluronic Acid (HA) Gels on Glass Substrates. Bacterial HA from Streptococcus zooepidemicus is mostly dissociated at neutral $\mathrm{pH}$, exhibiting a linear charge density of 0.7 charges/monomer unit. ${ }^{6}$ The polymer was anchored on glass cover slides $\left(24 \times 24 \times 0.1 \mathrm{~mm}^{3}\right)$ through aminosilane monolayers. The monolayers of AHAPTMS were deposited following a method derived from Haidara et al. ${ }^{7}$ Glass slides were first cleaned with "Piranha solution" $\left(30 \% \mathrm{H}_{2} \mathrm{O}_{2}\right.$ and $70 \% \mathrm{H}_{2} \mathrm{SO}_{4}$ ) for about $1 \mathrm{~h}$ and then rinsed extensively with water. They were then immersed overnight into an absolute ethanol solution containing $1 \mu \mathrm{L} \mathrm{ml} \mathrm{m}^{-1}$ AHAPTMS 95\%. The glass slides were extensively rinsed with absolute ethanol and water and dried with a nitrogen flux before use. Measuring chambers with the silanized substrates as bottom were assembled. For this purpose, each silanized glass slide was glued with silicone grease (Wacker, Munich, Germany) to one side of a square Teflon frame (outer side dimension $24 \mathrm{~mm}$ and height $2 \mathrm{~mm}$ ) (Figure 1). HA powder was dissolved in $10 \mathrm{mM}$ HEPES (pH 7.0) buffer at a concentration of 2, 3, or $4 \mathrm{mg} \mathrm{ml}^{-1}$. Cross-linking and surface-grafting of HA was achieved by dissolving 0.05 M NHS and 0.2 M EDC in the HA solution. After an incubation period of $5 \mathrm{~min}$, the HA/EDC/NHS solution was deposited onto the silanized glass slides. Variation of the amount of polymer solution deposited on the substrates allowed us to vary the thickness of gels from about $200 \mu \mathrm{m}$ to $2 \mathrm{~mm}$. Gelation was completed after about $4 \mathrm{~h}$. Then the chambers, which were open at the top, were stored in small glass flasks filled with about $5 \mathrm{~mL}$ water.

Coupling of Magnetic and Nonmagnetic Beads to the Surface of HA Gels. The density of beads grafted onto the surface of the gels was controlled by gently shaking the flasks containing the HA-covered substrates overnight on an incubator table with known amounts of magnetic and nonmagnetic beads added to the solution. The amino-functionalized nonmagnetic beads are coupled to the surface of activated HA gels either through simple adsorption or more likely through amide bonds. Tosyl-activated magnetic beads are covalently bound to the gel surface through a nucleophilic substitution reaction with the carboxylate or alcohol groups of HA. Gentle rinsing of the sample with water prior to observation allowed removal of unbound particles. Figure 2 shows a schematic view of the surface-grafted HA gels.

Magnetic Tweezers Setup. The measuring chamber and the magnet have been described in detail previously. ${ }^{8,9}$ The magnetic

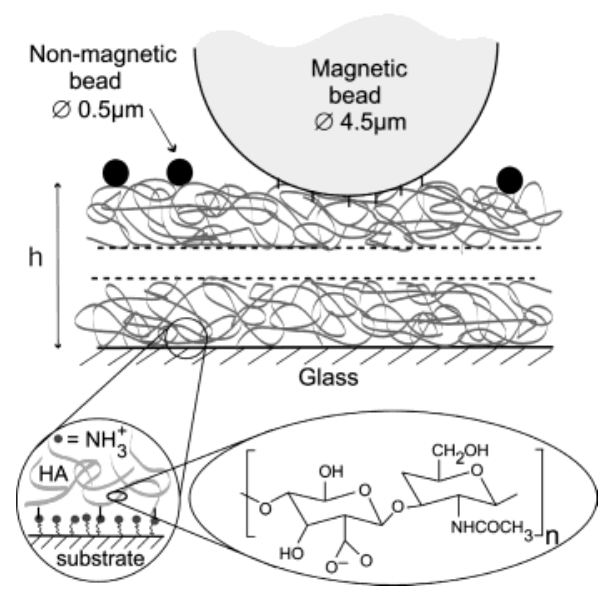

Figure 2. Schematic view of the hyaluronic acid (HA) gel covalently grafted to a coverglass with a magnetic probe and nonmagnetic colloidal beads grafted onto the surface. The thickness, $h$, may vary between $200 \mu \mathrm{m}$ and $2 \mathrm{~mm}$. Note that the drawing is not to scale.

field is generated by a solenoid with a soft iron core exhibiting a wedge-shaped nose (Figure 1). The nose penetrates the measuring chamber and the distance between the magnetic probes and the tip of the iron core can be adjusted with an accuracy of a few micrometers. Depending on the distance between the bead and the coil, forces of up to several hundred nanonewtons may be generated. The measuring cell consists of an aluminum block into which a $24 \times 24 \mathrm{~mm}^{2}$ Teflon frame is fitted, which serves as a holder for the glass slides supporting the HA gels. A function generator FG 9000 (ELV, Leer, Germany) triggers a custom-built amplifier to activate the magnetic solenoid. The magnet and the measuring chamber are mounted on the stage of an Axiovert 100 microscope (Zeiss, Oberkochen, Germany). For the observation of the magnetic and nonmagnetic beads, phase contrast images are taken with a Zeiss LD Achroplan objective $(\times 32 / 0.75 \mathrm{Ph} 2)$ and a CCD camera (C4880-80, Hamamatsu Photonics, Hamamatsu City, Japan) connected to a numerical data acquisition system. The position of the particles is determined with an accuracy of $\sim 10$ nm using a self-written single particle tracking algorithm implemented in the public domain image processing software from the National Institutes of Health Image (National Institutes of Health, Bethesda, MD). The thickness of the HA gels at room temperature was estimated by moving the focus of the objective from the glass surface to the surface of the gel.

Cell Adhesion. We studied the adhesion of two types of cells, mammalian 3T3 fibroblasts (NIH, Bethesda, MD) and cells of the slime mold Dictyostelium discoideum (wild-type AX2). For 3T3 fibroblast adhesion, HA gels were first coated with fibronectin by incubating the samples overnight in a $5 \mu \mathrm{g} \mathrm{ml}^{-1}$ fibronectin solution. The 3T3 fibroblasts were then cultured overnight onto the gels in an incubator at $37{ }^{\circ} \mathrm{C}$ and $5 \% \mathrm{CO}_{2}$. The cell culture medium consisted of Dulbecco's modified Eagle medium (DMEM) with 10\% v/v fetal calf serum (Life Technologies, Frederick, MD). The experiments were conducted at room temperature. Cells of Dictyostelium discoideum were cultivated on a Petri dish in liquid nutrition medium (from MaxPlanck-Institut fur Biochemie, Martinsried, Germany). Prior to each experiment, cells were detached from the bottom of the Petri dish by gentle hydrodynamic flow of the medium. The suspended cells were then deposited onto the HA-covered substrate mounted in the measurement cell on the microscope stage. 

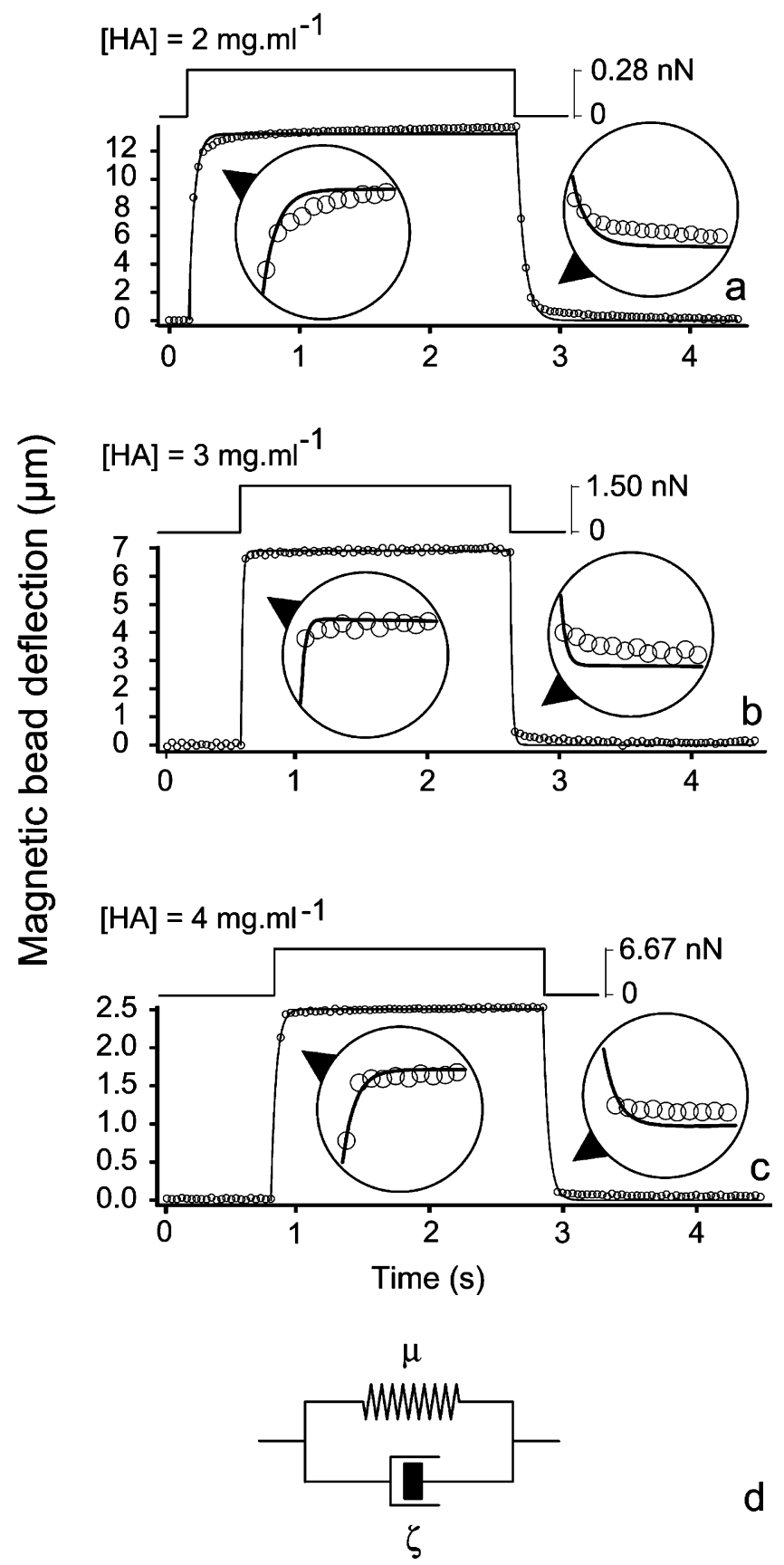

d

Figure 3. Viscoelastic response curves of HA gels of various concentrations: (a) 2; (b) 3; (c) $4 \mathrm{mg} \mathrm{ml}^{-1}$. The circles represent the measured deflection of the magnetic probes in the tangential direction induced by force pulses of $2 \mathrm{~s} \mathrm{(b}$ and $\mathrm{c}$ ) and $2.5 \mathrm{~s}$ (a) duration. Force pulses are represented above each curve. The measured creep responses were analyzed with a Voigt body (d) of frictional coefficient $\zeta$ and spring constant $\mu$. Full lines correspond to the optimal fits. Note that the force amplitude is different for each example.

\section{Results and Discussion}

Viscoelasticity of the Gels. Creep response experiments were performed to determine the viscoelastic nature of the gels. Magnetic beads grafted onto the surface were displaced tangentially by application of force pulses of known amplitude. The displacements of the beads on the surface of the gels were measured by tracking their positions with the software described above. Figure 3 shows three typical creep response experiments for HA gels of concentrations 2,3 , and $4 \mathrm{mg} \mathrm{ml}^{-1}$. It is seen that the deflection of the magnetic probe always saturates and completely relaxes to its initial position after switching off the magnetic field. This shows that the HA gels behave as perfect viscoelastic solids.

We tried to account for the viscoelastic response in terms of the most simple mechanical equivalent circuit consisting of a parallel array of a dashpot (with frictional coefficient $\xi$ ) and an elastic spring (of spring constant $\mu$ ) called a Voigt body 8 (Figure 3d). The deflection, $x(t)$, in the tangential direction as a function of time can be expressed as

$$
x(t)=\frac{f_{0}}{\mu}\left\{1-\exp \left(-\frac{t}{\tau}\right)\right\}
$$

where $f_{0}$ is the force and $\tau$ is the relaxation time. The relaxation time is related to the frictional coefficient as $\tau=\zeta / \mu ; \mu$ is an effective elastic modulus with dimension $\mathrm{N} \mathrm{m}^{-1}$. It can be considered as an effective surface shear modulus, which can be related to the bulk shear modulus $G$ or the Young modulus $E$, respectively, to a first approximation by

$$
G=k \frac{\mu}{a}
$$

and ${ }^{5}$

$$
E=2(1+v) G=k \frac{2 \mu(1+v)}{a}
$$

where $v$ is the Poisson ratio and $a$ is the radius of contact between the bead and the polymer surface. The parameter $k$ is a coefficient the determination of which will be discussed below. Analysis of the response curves yields the values reported in Table 1 for the viscoelastic parameters $\mu, \tau$, and $\xi$ of the three examples shown in Figure 3.

To determine the bulk shear modulus $G$ (or Young modulus $E$ ) and the bulk viscosity of the HA network, the contact area between the bead and the gel surface must be known. Because this parameter cannot be measured directly, a different method to determine the Young modulus and Poisson ratio $v$, "the deformation field technique", is applied below.

Deformation Field Analysis. In the following, we describe the determination of the elastic modulus of the HA networks by a previously developed deformation field analysis. For this purpose, the surface of the HA gel is decorated with colloidal beads of $0.5 \mu \mathrm{m}$ diameter together with a few paramagnetic beads at a ratio of 10 000:1. Tangential force pulses of severalpiconewton amplitudes were applied, and the deflection of the nonmagnetic colloidal probes was determined yielding the induced tangential deflection, $u(r)$, as a function of the distance of the colloidal probes from the center of the force. The deformation field can be analyzed by application of the theory of elastic deformation of an elastic body limited by a plane by a pointlike force. Following Landau and Lifschitz, ${ }^{5}$ the displacement $\mathbf{u}\left(u_{x}, u_{y}\right)$ of a point $\mathbf{P}(x, y)$ induced by a force $\mathbf{F}\left(F_{x}, 0\right)$ applied at position $\mathbf{P}_{0}(0,0)$ is given by

$$
u_{x}=\frac{1+v}{\pi E} \frac{1-v+\frac{v x^{2}}{x^{2}+y^{2}}}{\sqrt{x^{2}+y^{2}}} F_{x}
$$

and

$$
u_{y}=\frac{1+v}{\pi E} \frac{v x y}{\left(x^{2}+y^{2}\right)^{3 / 2}} F_{x}
$$

where $v$ is the Poisson ratio and $E$ is the Young modulus. 
TABLE 1: Summary of the Viscoelastic Parameters Obtained by Analysis of Creep Response Curves in Terms of the Voigt Model (Figure 3) for the Three HA Gels Studied

\begin{tabular}{|c|c|c|c|c|c|c|}
\hline & \multicolumn{2}{|c|}{$[\mathrm{HA}]=2 \mathrm{mg} \mathrm{ml}^{-1}$} & \multicolumn{2}{|c|}{$[\mathrm{HA}]=3 \mathrm{mg} \mathrm{ml}^{-1}$} & \multicolumn{2}{|c|}{$[\mathrm{HA}]=4 \mathrm{mg} \mathrm{ml}^{-1}$} \\
\hline & extension & relaxation & extension & relaxation & extension & relaxation \\
\hline$\mu\left(\mathrm{N} \mathrm{m}^{-1}\right)$ & $2.1 \times 10^{-5}$ & $2.1 \times 10^{-5}$ & $2.2 \times 10^{-4}$ & $2.2 \times 10^{-4}$ & $2.6 \times 10^{-3}$ & $2.6 \times 10^{-3}$ \\
\hline$\tau(\mathrm{s})$ & 0.042 & 0.058 & 0.011 & 0.014 & 0.037 & 0.040 \\
\hline$\zeta\left(\mathrm{N} \mathrm{s} \mathrm{m}^{-1}\right)$ & $8.8 \times 10^{-7}$ & $12.2 \times 10^{-7}$ & $2.4 \times 10^{-6}$ & $3.1 \times 10^{-6}$ & $9.6 \times 10^{-5}$ & $10.5 \times 10^{-5}$ \\
\hline
\end{tabular}
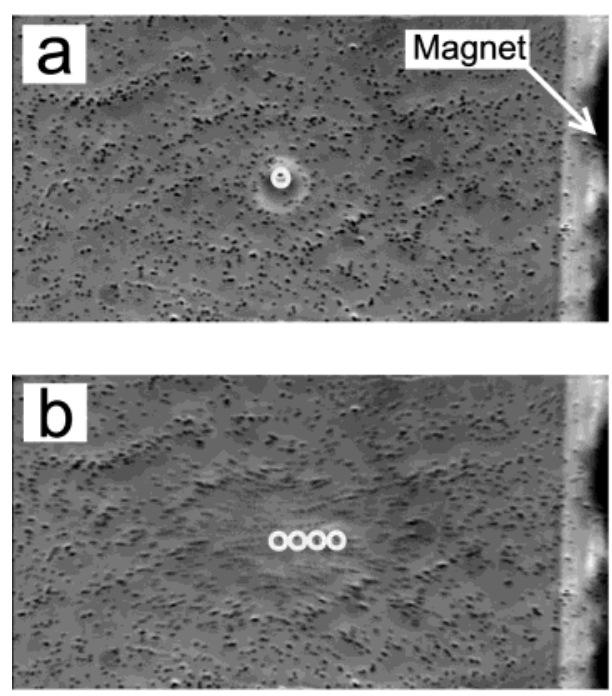
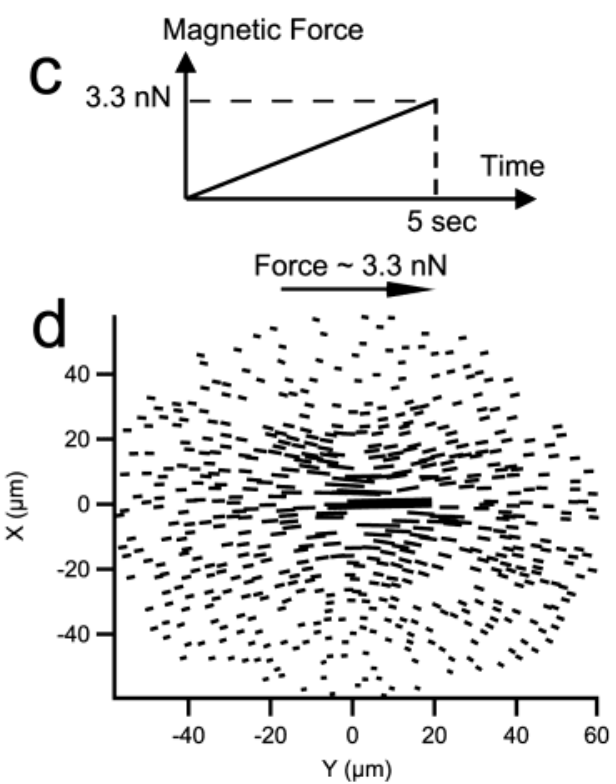

Figure 4. Example of deformation field measurement of a $\mathrm{HA}$ gel of $2 \mathrm{~mm}$ thickness and $3 \mathrm{mg} \mathrm{ml}^{-1}$ polymer concentration. Images taken by phase contrast microscopy. Panel a shows the distribution of magnetic and nonmagnetic probes at rest. The contour of the magnetic bead is indicated by a white circle. Panel b shows the superposition of four snapshots taken during a sawtooth-like force pulse. The force (panel c) increases linearly from 0 to $3.3 \mathrm{nN}$ within $5 \mathrm{~s}$. Panel d shows the trajectory of colloidal beads induced by sawtooth-like pulses determined by particle tracking.

We measured the displacements $u_{x}$ and $u_{y}$ of up to 500 nonmagnetic beads in the neighborhood of the point of application of the force $F_{x}$. Figure 4 gives an example of such an experiment of "strain-field mapping" for a $2 \mathrm{~mm}$ thick gel deposited from a $3 \mathrm{mg} \mathrm{ml}^{-1} \mathrm{HA}$ solution onto a glass slide. Figure $4 \mathrm{a}$ shows the initial equilibrium state of this gel. Figure $4 \mathrm{~b}$ presents a superposition of successive bead positions when the force applied onto the magnetic probe increases linearly as shown in Figure 4c. Figure 4d shows the deflection amplitudes $\mathbf{u}[\mathbf{P}(x, y)]$ of the surface reconstructed by tracking all nonmagnetic particles surrounding the magnetic bead. This deformation field agrees well with that predicted by eqs 3 and 4 . The same deformation field was observed for all other HA concentrations. The deformation fields extend over $60 \mu \mathrm{m}$, which shows that the polymer gel is homogeneous at least over this lateral dimension.

The Poisson ratio was determined from the amplitude ratio $u_{x} / u_{y}$ according to

$$
v=\frac{x^{2}+y^{2}}{x y\left(u_{x} / u_{y}\right)+y^{2}}
$$

This equation is obtained by combining eqs 3 and 4. By measuring $x, y, u_{x}$, and $u_{y}$ for every nonmagnetic bead, it was possible to determine the local values of the Poisson ratio of the gel. The large number of beads evaluated for each deflection experiment allows the statistical analysis of $v$ in terms of distribution functions. An example of such a distribution function of Poisson ratio's is shown in the inset b of Figure 5. The distribution of $v$ was fitted with a Gaussian curve. Figure 5a shows the experimental values of $v$ obtained by this statistical

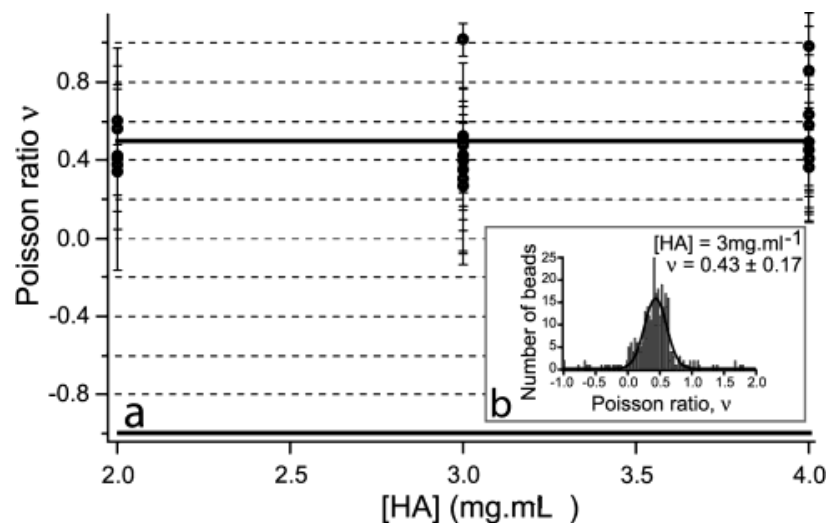

Figure 5. Poisson ratios, $v$ (a), measured for three HA gels of different concentrations. The inset $\mathrm{b}$ shows an example of a Gaussian fit (full line) to the distribution of $v$ obtained for a $3 \mathrm{mg} \mathrm{ml}^{-1}$ gel by analyzing the deflection of the colloidal bead with eq 4 . The circles and error bars given in panel a are, respectively, the mean values and the standard deviations given by the Gaussian fits.

method for the three different HA concentrations. For each measurement of $v$ (circles) in Figure 5a, the standard deviation was determined, and the value obtained is given as vertical bar. As demonstrated in Figure 5a, the concentration of HA has no influence on the values of $v$. Most of the samples were found to exhibit Poisson ratios between 0.3 and 0.5 meaning that the gels are almost incompressible but very soft with respect to shearing. This is because HA is a very hydrophilic molecule known as a "molecular sponge" that takes up a large amount of water.

Some samples ( 8 out of 40) were more puzzling because they exhibited Poisson ratios larger than 0.5 , which is theoretically 

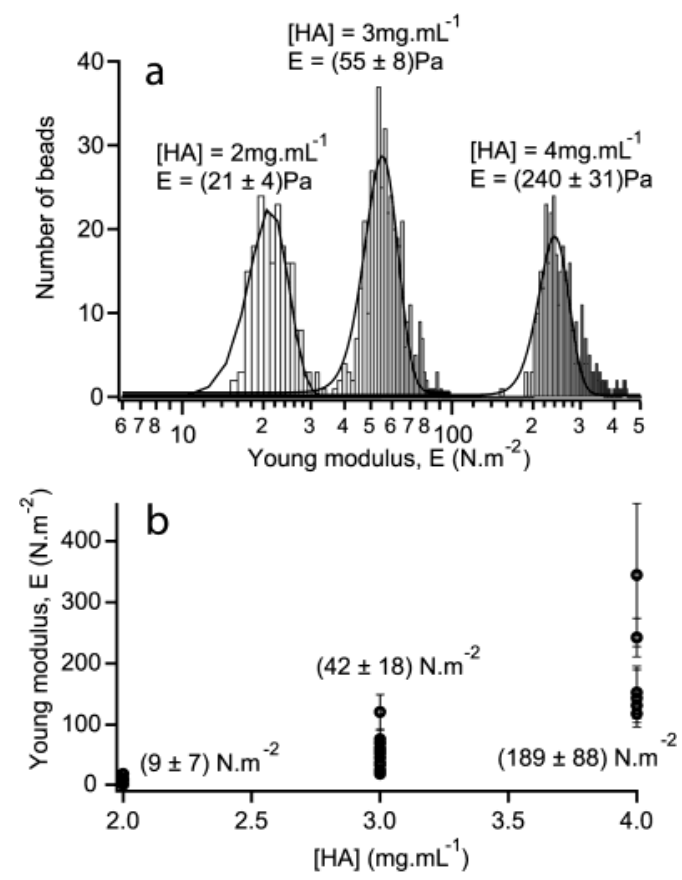

Figure 6. Statistical determination (a) of Young moduli $E$ for three HA gels of different concentrations. Full lines are Gaussian fits to the distribution of $E$ obtained with eq 3 for every nonmagnetic bead tracked. Note that the Gaussian profile is distorted by the logarithmic scale. Panel b provides a summary of the values obtained by Gaussian fitting for various HA gels of different concentrations. Error bars are the standard deviations determined from the Gaussian fits. The values of $E$ indicated are the mean Young moduli and the standard deviation found for each concentration.

TABLE 2: Estimation of the Contact Radius $a$ between the Magnetic Bead and the HA Surface for the Three Gels Studied $^{a}$

\begin{tabular}{lccc}
\hline & {$[\mathrm{HA}]=2 \mathrm{mg} \mathrm{ml}^{-1}$} & {$[\mathrm{HA}]=3 \mathrm{mg} \mathrm{ml}^{-1}$} & {$[\mathrm{HA}]=4 \mathrm{mg} \mathrm{ml}^{-1}$} \\
\hline$\mu\left(\mathrm{N} \mathrm{m}^{-1}\right)$ & $2.1 \times 10^{-5}$ & $2.2 \times 10^{-4}$ & $2.6 \times 10^{-3}$ \\
$E\left(\mathrm{~N} \mathrm{~m}^{-2}\right)$ & $9 \pm 7$ & $42 \pm 18$ & $189 \pm 88$ \\
$a(\mu \mathrm{m})$ & 0.8 & 1.9 & 4.9
\end{tabular}

${ }^{a}$ The values are obtained by comparison of spring constants measured by the viscoelastic experiment and the averages of the Young modulus obtained by the deformation field mapping technique.

impossible because $v$ can vary only between -1 for nondeformable and 0.5 for incompressible elastic solids. We ignored these few samples for the following determination of the Young moduli. The samples with $-1<v<0.5$ were further evaluated by inserting $v$ into eq 3 and determining the local values of the Young modulus $E$. We chose eq 3 rather than eq 4 because the deflection $u_{x}$ in the force direction $x$ can be measured more accurately than that of $u_{y}$. The analysis of the deflection of the whole population of nonmagnetic beads enabled us to determine the distributions of the Young moduli $E$. In Figure 6a, examples of the distributions of $E$ obtained in this way are presented for the three HA concentrations showing the increase in the elastic modulus. For each sample, the distributions were fitted with a Gaussian curve to determine the values of $E \pm \sigma$, where $\sigma$ is the standard deviation. Figure $6 \mathrm{~b}$ summarizes all of the values measured, and the standard deviations are again indicated as vertical bars. These results are also summarized in Table 2 .

The elastic modulus increases astonishingly strongly with the polymer concentration [HA]. According to Table 2, a power law $E \approx[\mathrm{HA}]^{3.5}$ holds, in contrast with the power law predicted for flexible polymers: $:^{10} E \approx[\mathrm{HA}]^{2.25}$. One possible explanation for this anomalous increase of the elastic modulus with HA concentration is the associated increase of ionic strength. Judged from experiments by Albersdörfer and Sackmann, ${ }^{11}$ the elastic modulus at constant polymer concentration increases roughly linearly with ionic strength (between 20 and $50 \mathrm{mM}$ ). This suggests a power law $\sim[\mathrm{HA}]^{3}$ for strongly charged entangled polyelectrolytes. Nothing is known yet about the concentration dependence of the elasticity of cross-linked polyelectrolytes.

Although the strain field mapping technique is the most rigorous approach to determine the elastic moduli (e.g., $E$ ) of the polymer films, for most practical purposes, it is too cumbersome. It is more convenient to determine the Young modulus approximately from viscoelastic experiments by making use of eq $2 \mathrm{~b}$. The prefactor can be obtained from eq 3 by assuming that the shear stress generated by the force probe can be represented by a point force at position $x=0, y=0$ and that this force induces the same deformation field as the distributed force. By considering the deformation at the position $(a, 0)$ and $(0, a)$, we obtain (for $v=1 / 2)$ for the prefactor $k_{1}=$ $1 /(2 \pi)$ and $k_{2}=1 /(4 \pi)$, respectively. At the present state of approximation, it is reasonable to take the arithmetic mean of the two values of $k_{i}$, which is $k=3 /(8 \pi)$, and write $E=(9 /$ $(8 \pi)) \mu / a$.

To test the validity and the accuracy of this approach, we calculate the radius of contact $a$ by inserting the value of $E$ obtained by statistical analysis (Figure 6). The values of $a$ presented in Table 2 agree within a factor of 2 with the radius of the bead. Considering the fact that the bead will form an indentation at the polymer surface due to the gravitational force of the magnetic bead and that it is covalently coupled to the HA, this agreement is considered as satisfactory. The simplified procedure to measure the Young modulus by a viscoelastic response experiment yields thus values of satisfactory accuracy for most practical applications.

Another possible approach to estimate the contact radius $a$ is to calculate the radius of the indentation of the gel substrate caused by the gravitational force by application of the Hertz model. ${ }^{5}$ With the Young moduli of Figure 6, one obtains values of $a$ of $0.3,0.22$, and $0.15 \mu \mathrm{m}$ for the three polymer densities 2 , 3, and $4 \mathrm{mg} \mathrm{ml}^{-1}$, respectively. The values of the Young moduli obtained by analysis of the viscoelastic response curves are larger by over an order of magnitude than the measured data. This discrepancy is attributed to the fact that the contact area is determined by the covalent coupling of the beads to the polymer surface and that the bead penetrates therefore deeper into the gel than it would by pure gravitational force.

Behavior of Submicrometer Thick Gels. The deformation behavior of thin polymer films (200-800 $\mu \mathrm{m}$ thickness) differs remarkably from that of the thick layers as demonstrated in Figure 7 where we present the deformation field of a $530 \mu \mathrm{m}$ thick, $2 \mathrm{mg} \mathrm{ml}^{-1} \mathrm{HA}$ gel as induced by a force increasing linearly with time as shown in Figure 7c. Figure 7a shows the initial distribution of the beads, while in Figure $7 \mathrm{~b}$ the superposition of successive positions of the probe beads representing the trajectories are shown. The trajectories measured by particle tracking are represented in Figure 7d, which shows that the deformation field exhibits a vortex structure.

The circular motions of the colloidal probes can be rationalized in terms of the nonlinear viscoelastic response of the polymeric fluid. ${ }^{12,13}$ First it should be noted that the shear deformation is larger by a factor of 10 in the case of the thin layers $\left(\gamma_{x z} \approx 10^{-2}\right)$ than in the case of the thick layer $\left(\gamma_{x z} \approx\right.$ $\left.10^{-3}\right)$. It is a well-known rule that polymers in a flow are deformed in such a way that they generate a tension in the direction of the flow line and that this behavior gives rise to nonlinear viscoelastic behavior. One consequence is the genera- 

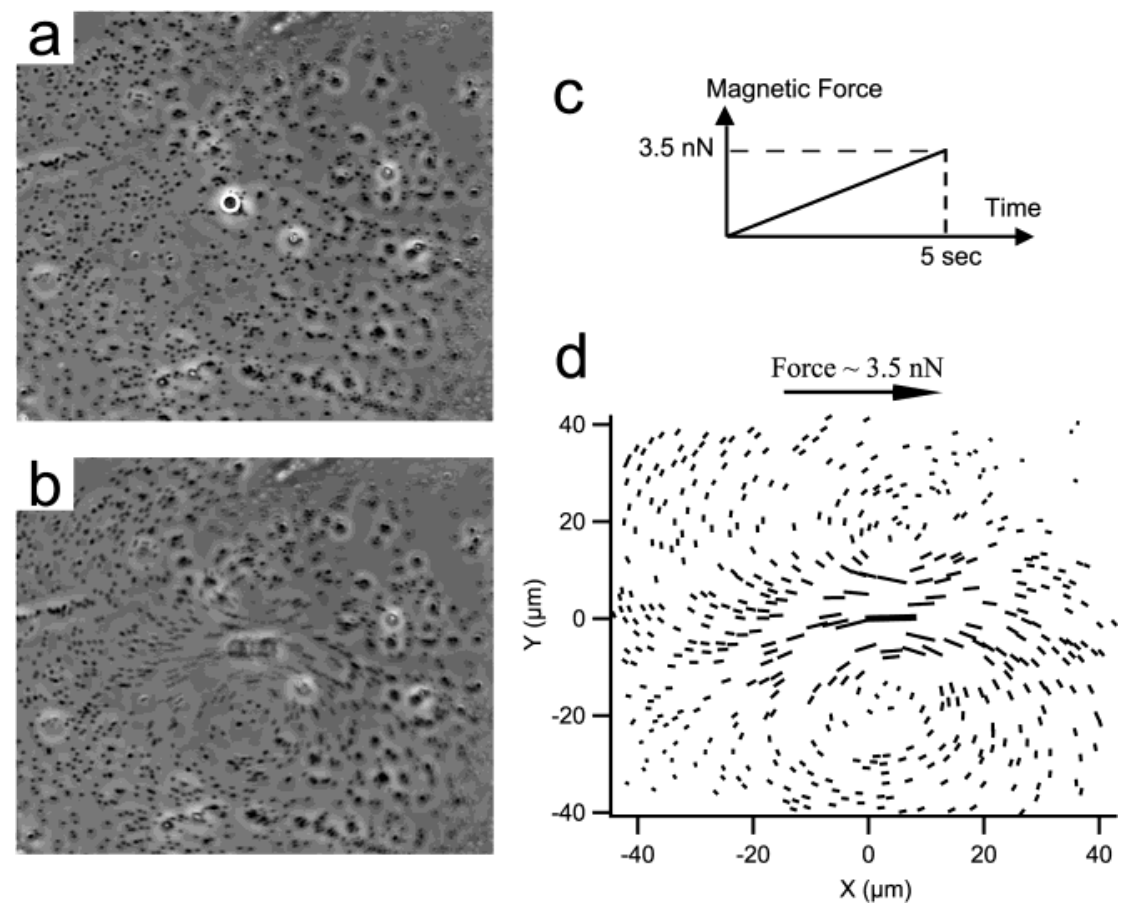

Figure 7. Example of surface deformation field measurement of HA gel of $530 \mu \mathrm{m}$ thickness and $2 \mathrm{mg} \mathrm{ml}^{-1}$ polymer concentration. Images taken by phase contrast microscopy. Panel a shows the distribution of magnetic and nonmagnetic probes at rest. The contour of the magnetic bead is indicated by a white circle. Panel $b$ shows the superposition of four snapshots taken during a sawtooth-like force pulse. The force (panel $c$ ) increases linearly from 0 to $3.5 \mathrm{nN}$ within $5 \mathrm{~s}$. Panel d shows the trajectory of colloidal beads determined by particle tracking.
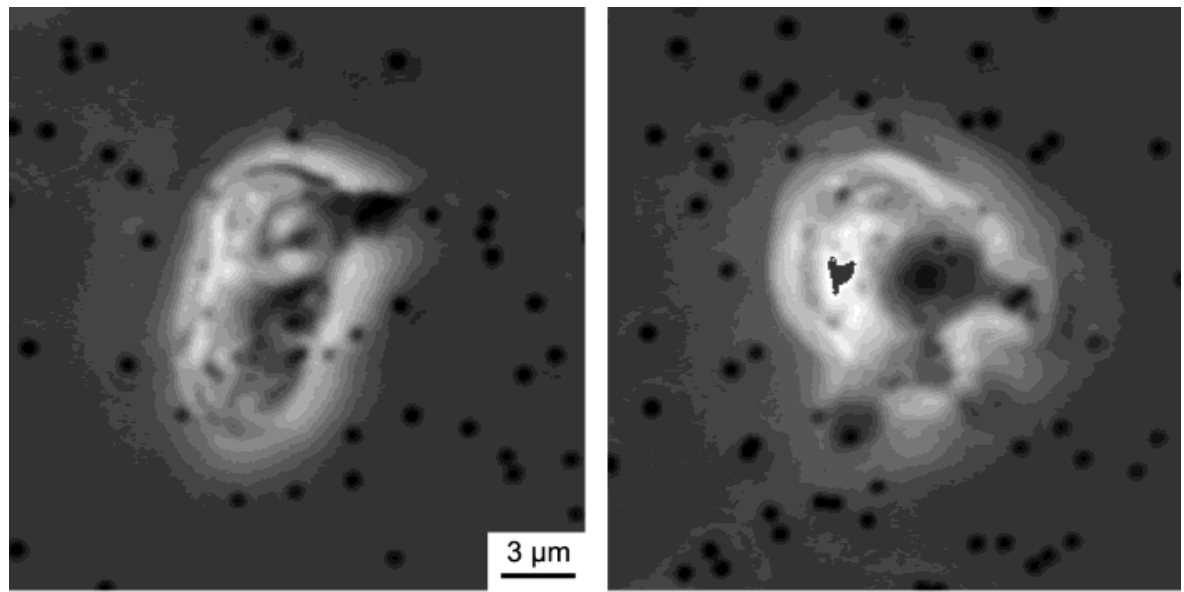

Figure 8. Cells of Dictyostelium discoideum adhering onto a $4 \mathrm{mg} \mathrm{ml}^{-1} \mathrm{HA}$ gel decorated with $500 \mathrm{~nm}$ colloidal beads (dark points on the image). One can notice numerous protrusions made by the cells, which deform the gel surface by protrusion and retraction forces.

tion of normal stresses, which are proportional to the square of the shear rate: $N=\phi(\mathrm{d} \gamma / \mathrm{d} t)^{2}{ }^{12}$ For a particle on the $x$-axis of the coordinate system with the $x$-axis parallel to the flow, it is $N=\sigma_{x x}-\sigma_{y y}$. In our case, the tensions have different consequences for the polymers in the positive $(+x)$ and negative $(-x)$ half-planes. They are stretched parallel to the force direction for positions $x<0$ and compressed for $x>0$. This is expected to give rise to a tension gradient in the angular direction ( $r=$ const), which decreases in the negative $x$-direction. This dynamically induced tension gradient will result in a backflow of the polymer near the surface. The fact that the backflow appears below a threshold value of the HA-layer thickness (and thus above a limiting value of the shear rate) provides evidence for the explanation in terms of the nonlinear viscoelasticity.

Adhesion of Cells onto the HA Gels. Because one motivation for the present study was to generate models of bioanalogous tissue surfaces for future studies of cell adhesion or cell locomotion on soft surfaces of well-defined viscoelastic proper- ties, we tested the adhesion properties of different cells on the HA films. Figure 8 shows examples of cells of Dictyostelium discoideum adhered on a $4 \mathrm{mg} \mathrm{ml}^{-1} \mathrm{HA}$ gel, while Figure 9 shows examples of $3 \mathrm{~T} 3$ fibroblasts cultured onto a $2 \mathrm{mg} \mathrm{ml}^{-1}$ HA gel. The two cells behave remarkably different. While Dictyostelia adhere readily on pure HA layers also in the absence of the grafted beads, fibroblasts do not. They hover over the surface avoiding any contact unless the HA surface is functionalized by adsorption of fibronectin or tosyl-activated Dynabeads covered by fibronectin or both. Remarkably, the fibroblasts tend to stretch between the beads suggesting that they adhere only weakly on the soft HA-film.

\section{Concluding Remarks}

We showed that soft homogeneous films of hyaluronic acid of more than $200 \mu \mathrm{m}$ thickness can be covalently grafted onto glass substrates and that their viscoelastic moduli can be 

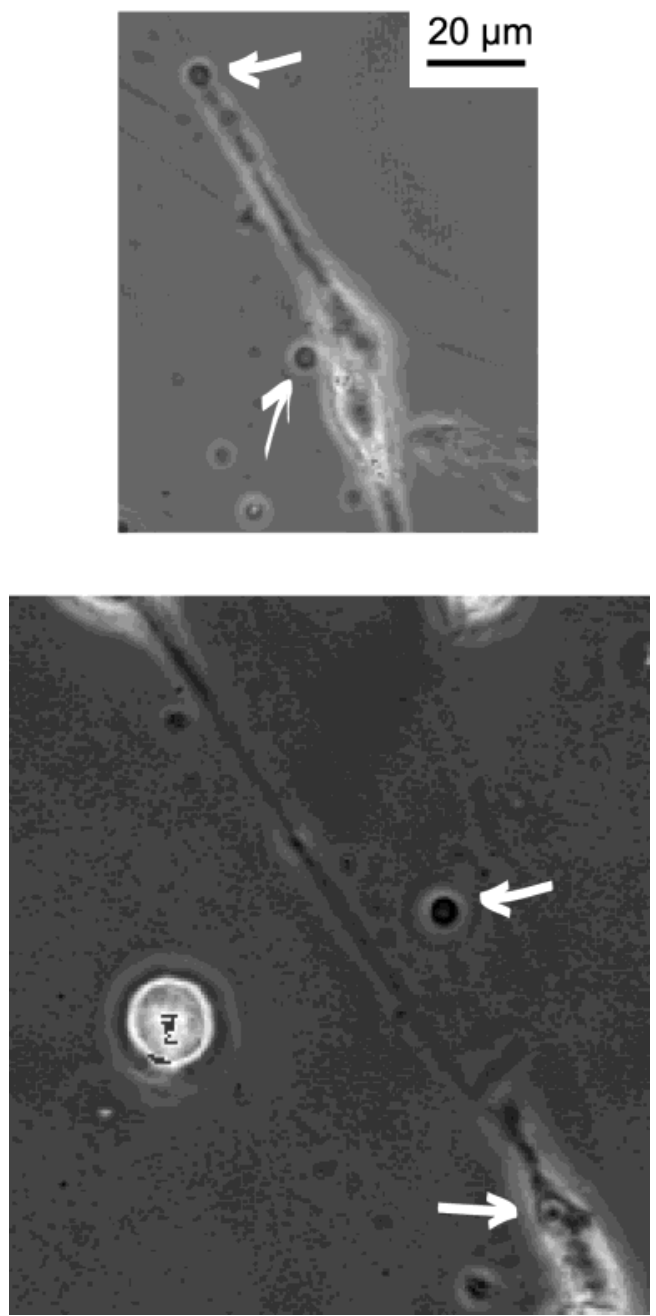

Figure 9. Example of $3 \mathrm{~T} 3$ fibroblasts adhering onto a $2 \mathrm{mg} \mathrm{ml}^{-1} \mathrm{HA}$ gel that was first decorated with tosyl-activated Dynabeads and then coated with fibronectin. White arrows indicate position of magnetic beads of diameter $4.5 \mu \mathrm{m}$ grafted to the surface of the gel.

manipulated by chemical cross-linking without loss of the homogeneity. Homogeneously cross-linked films thinner than $200 \mu \mathrm{m}$ could not be generated yet. The main motivation of the work was to generate bioanalogous mimetics of tissue surfaces for future measurements of protrusion forces generated by pseudopods, adhesion forces following Simson et al., ${ }^{14}$ and traction forces associated with moving cells following the work of other groups. ${ }^{15-17}$ Because for such measurements the viscoelastic properties of the soft surfaces have to be known, we developed micromechanical techniques (i) to measure the surface shear modulus, the surface viscosity, and the bulk Young modulus and (ii) to characterize the homogeneity of the gel films.

Owing to its huge size (molecular weights are on the order of $10^{6} \mathrm{Da}$ ), HA enables the generation of very soft polymer cushions exhibiting elastic moduli that are softer by $2-4$ orders of magnitude than those of polyacrylamide gels. This allows the measurement of weaker forces between cells and substrates such as the forces generated by single advancing pseudopods of Dictyostelia cells. These results will be presented in a forthcoming paper.

Acknowledgment. The work was supported by the Deutsche Forschungsgemeinschaft (Grant SFB 413) and the Fonds der Chemicher Industrie. Laurent Vonna acknowledges the fellowship received by the Alexander von Humboldt Foundation and the European Community (Grant MCFI-1999-00252).

\section{References and Notes}

(1) Sackmann, E.; Bruinsma, R. F. ChemPhysChem 2002, 3, 262.

(2) Zimmerman, E.; Geiger, B.; Addadi, L. Biophys. J. 2002, 82, 1848.

(3) Toole, B. P. In Cell Biology of Extracellular Matrix, 2nd ed.; Hay, E. D., Ed.; Plenum Publishing Corp.: New York, 1992.

(4) Sengupta, K.; Schilling, J.; Marx, S.; Fischer, M.; Bacher, A.; Sackmann, E. Langmuir 2003, 19, 1775.

(5) Landau, L. D.; Lifshitz, E. M. Theory of Elasticity; Pergamon Press: London, 1959. 3710 .

(6) Rinaudo, M.; Milas, M.; Jouon, N.; Borsali, R. Polymer 1993, 34,

(7) Haidara, H.; Mougin, K.; Schultz, J. Langmuir 2000, 16, 7773.

(8) Ziemann, F.; Rädler, J.; Sackmann, E. Biophys. J. 1994, 66, 2210.

(9) Bausch, A. R.; Ziemann, F.; Boulbitch, A. A.; Jacobson, K. Sackmann, E. Biophys. J. 1998, 75, 2038.

(10) De Gennes, P.-G. Scaling Concepts in Polymer Physics; Cornell University Press: Ithaca, NY, 1979.

(11) Albersdörfer, A.; Sackmann, E. Eur. Phys. J. B 1999, 10, 663.

(12) Barnes, H. A.; Hutton, J. F.; Walters, K. An Introduction to Rheology; Elsevier: Amsterdam, 1989; Chapter 4.

(13) Bird, R. B.; Armstrong, R. C.; Hassager, O. Dynamics of Polymeric Liquids, Vol. 1, Fluid Dynamics; Wiley: New York, 1987.

(14) Simson, D. A.; Ziemann, F.; Strigl, M.; Merkel, R. Biophys. J. 1998 74, 2080.

(15) Lee, J.; Leonard, M.; Oliver, T.; Ishihara, A.; Jacobson, K. J. Cell Biol. 1994, 127, 1957.

(16) Oliver, T.; Jacobson, K.; Dembo, M. Cell Motil. Cytoskeleton 1995, 31,225

(17) Balaban, N. Q.; Schwarz, U. S.; Riveline, D.; Goichberg, P.; Tzur, G.; Sabanay, I.; Mahalu, D.; Safran, S.; Bershadsky, A.; Addadi, L.; Geiger, B. Nature Cell Biol. 2001, 3, 466. 